\title{
MEPHISTO AMSTERDAM FIRST IN SWEDISH RATING LIST
}

\author{
ICCA Communication \\ by Göran Grottling
}

The new rating list, based on 4358 games played at tournament level, is very similar to the previous one (ICCA Journal, Vol. 9, No. 2, p.113). Two new computers have made their entrance : Par Excellence from Fidelity and Constellation Quattro from Novag (2nd and 13th place). Mephisto Exclusive $S$ has been removed because of its obsoleteness. Any still around will have been converted to Mephisto Amsterdam. In the list below the figures in parentheses indicate the number of games recorded.

\begin{tabular}{r|l|r|r|}
\hline 1. & Mephisto Amsterdam 12 MHz & 2026 & $(448)$ \\
2. & Avant Garde 5 MHz & 1932 & $(382)$ \\
4. & Par Excellence 5 MHz & 1932 & $(120)$ \\
5. & Excellence 4 MHz & 1895 & $(310)$ \\
6. & Expert 4 MHz & 1891 & $(287)$ \\
7. & Conchess Plymate 4 MHz & 1871 & $(464)$ \\
8. & Excellence 3 MHz & 1850 & $(323)$ \\
9. & Turbostar 432 4 MHz & 1839 & $(458)$ \\
10. & Elegance 3.6 MHz - Private Line & 1837 & $(610)$ \\
11. & Mephisto MM 2 3.7 MHz & 1824 & $(422)$ \\
12. & Super Constellation 4 MHz & 1809 & $(231)$ \\
13. & Conchess Glasgow 4 MHz & 1794 & $(1004)$ \\
14. & Constellation Quattro 4 MHz & 1776 & $(448)$ \\
15. & Mephisto B\&P 3.7 MHz & 1776 & $(68)$ \\
16. & Superstar 36 K 2 MHz & 1772 & $(173)$ \\
17. & Prestige 4 MHz & 1744 & $(345)$ \\
18. & Conchess Glasgow 2 MHz & 1724 & $(280)$ \\
19. & Elite A/S 3.2 MHz - Sensory 12 & 1717 & $(378)$ \\
20. & Constellation 3.6 MHz & 1702 & $(470)$ \\
21. & Mephisto Mondial 1.8 MHz & 1699 & $(522)$ \\
22. & Super Enterprise - Advanced Star Chess & 1653 & $(159)$ \\
23. & Constellation 2 MHz & $(213)$ \\
24. & Turbo 16 K & 1623 & $(499)$ \\
& & & $(102)$ \\
\hline
\end{tabular}

The new Par Excellence runs on a $5 \mathrm{MHz}$ processor as does Avant Garde. It might seem more than a coincidence that the two machines have achieved exactly the same rating. Could it be that they contain the same program? This will prove when Par Excellence will have played more games. According to Novag the Quattro contains the Super Constellation program. However we doubt this; Super Constellation runs on $56 \mathrm{~K}$, whereas the Quattro runs on $16 \mathrm{~K}$. Our assumption is that the Quattro is a stripped Super Constellation program in which many of the special features (such as the KBNK endgame) have been removed.

Quattro's big brother, Constellation Forte, will appear on the list next time. Provided it contains the Expert program, its rating can be expected to be about 1890 . 


\section{Computers vs. Human Rating List}

The ratings of the above list were calibrated by the ratings of Swedish human players. The calibration is done by entering the computers in all kinds of tournaments and competitions in Sweden. So far thirteen of the computers on the above list have played 212 games against human players. The resulting ELO points are presented in the table below.

\begin{tabular}{|r|l|r|r|}
\hline 1. & Mephisto Amsterdam & 1980 & $(25)$ \\
2. & Plymate 4 MHz & 1953 & $(6)$ \\
3. & Plymate 5.5 MHz & 1879 & $(12)$ \\
4. & Conchess Glasgow 4 MHz & 1858 & $(23)$ \\
5. & Expert & 1839 & $(6)$ \\
6. & Super Constellation & 1807 & $(60)$ \\
7. & Par Excellence & 1789 & $(8)$ \\
8. & Elegance & 1785 & $(16)$ \\
9. & Constellation 3.6 MHz & 1779 & $(15)$ \\
10. & Turbostar 432 & 1759 & $(17)$ \\
11. & Elite A/S & 1720 & $(7)$ \\
12. & Conchess Glasgow 2 MHz & 1700 & $(11)$ \\
13. & Constellation 2 MHz & 1650 & $(6)$ \\
\hline
\end{tabular}

Due to the low number of games played between humans and computers the ratings above are not always reliable as can been seen from the apparent difference between Plymate $4 \mathrm{MHz}$ and Plymate 5.5 $M H z$. They have played too few games to make the tabulation trustworthy. It may be assumed that Plymate $5.5 \mathrm{MHz}$ is not weaker than Plymate $4 \mathrm{MHz}$. The result of Par Excellence lies 100 points lower than what we expect from inter-computer games.

\section{The Fifth Conference on Advances in Computer Chess}

Full details on this conference have been published in the ICCA Journal, Volume 9, No.2 (June 1986), page $116 \mathrm{ff}$.

We remind you that in order to qualify for a reduced participation fee your application and payment must have been received by the undersigned before December 1, 1986. Closing date for all applications is February 1, 1987 and for submitting papers is January 15, 1987.

We can now announce that the conference will be the theme of a special issue of Artificial Intelligence, an International Journal, published by North-Holland, Amsterdam, under the Guest Editorship of Hans Berliner and Don Beal to contain a selection of papers presented. Also, full proceedings will appear in book form, edited by Don Beal and to be published by North-Holland.

\section{J.J. van Oosterwijk Bruyn Hilversumseweg 6 1251 EX Laren The Netherlands}

[It is hoped to reproduce those parts of the conference's results that are of special interest to our readership in future issues of this Journal as well. - Ed.] 